\title{
Global stability analysis of a delayed HIV model with saturated infection rate
}

\author{
jaouad Danane ${ }^{\mathrm{a}}$ and Karam Allali ${ }^{\mathrm{b}}$ \\ Laboratory of Mathematics and Applications, \\ Faculty of Sciences and Technologies, University Hassan II \\ of Casablanca, PO Box 146, Mohammedia,Morocco
}

\begin{abstract}
In this paper, the global stability of a delayed HIV model with saturated infection rate infection is investigated. We incorporate two discrete delays into the model; the first describes the intracellular delay in the production of the infected cells, while the second describes the needed time for virions production. We also derive the global properties of this two-delay model as function of the basic reproduction number $R_{0}$. By using some suitable Lyapunov functions, it is proved that the free-equilibrium point is globally asymptotically stable when $R_{0} \leq 1$, and the endemic equilibrium point is globally asymptotically stable when $R_{0} \geq 1$. Finally, in order to support our theoretical findings we have illustrate some numerical simulations.
\end{abstract}

Keywords: delay model, HIV infection, viral dynamics, global stability, numerical simulation.

\section{Introduction}

The human immunodeficiency virus (HIV) is a virus that gradually weakens the immune system. It is considered as the main cause for several deadly diseases after the resulting acquired immunodeficiency syndrome (AIDS) is reached. With 36.7 million people living with HIV, 2.1 million people becoming newly infected by HIV and more than 1.1 million deaths annually, HIV becomes a major global public health issue [1]. For this reason, several mathematical models describing the dynamics of HIV infection have been developed and studied [2-6]. The basic of them was suggested in [2]:

$$
\left\{\begin{array}{l}
\dot{x}=\lambda-d_{1} x(t)-k_{1} x(t) v(t) \\
\dot{y}=k_{1} x(t) v(t)-d_{2} y(t) \\
\dot{v}=a y(t)-d_{3} v(t)
\end{array}\right.
$$

where $x, y$ and $v$ denote the concentration of uninfected $\mathrm{CD}^{+}{ }^{+} \mathrm{T}$ cells, infected $\mathrm{CD} 4^{+} \mathrm{T}$ cells and free virus, respectively. Susceptible host cells CD $4^{+} \mathrm{T}$ cells are produced at a rate $\lambda$, die at a rate $d_{1}$ and become infected by virus at a rate $k_{1} x v$. Infected cells die at a rate $d_{2}$. Finally, the free virus is produced by infected cells at a rate $a$ and decays at a rate $d_{3}$. In our model we propose to change the mass action rate in (1) by a saturated infection rate $\frac{k_{1} x(t) v(t)}{x(t)+v(t)}$, called the saturated mass action [7] which describes better the rate of

\footnotetext{
a e-mail: jaouaddanane@gmail.com

b e-mail: allali@hotmail.com
}

viral infection, then the viral infection model becomes

$$
\left\{\begin{array}{l}
\dot{x}(t)=\lambda-d_{1} x(t)-\frac{k_{1} x(t) v(t)}{x(t)+v(t)}, \\
\dot{y}(t)=\frac{k_{1} x(t) v(t)}{x(t)+v(t)}-d_{2} y(t) \\
\dot{v}(t)=a y(t)-d_{3} v(t),
\end{array}\right.
$$

with the initial conditions $x(0)=x_{0}, y(0)=y_{0}$ and $v(0)=v_{0}$. The theoretical analysis of the epidemic models [9-12] show that the time delay plays a crucial role in the dynamical properties of the HIV infection models, in order to study the influence of time delay on the spread of HIV, we have illustrated the following model:

$$
\left\{\begin{array}{l}
\dot{x}(t)=\lambda-d_{1} x(t)-\frac{k_{1} x(t) v(t)}{x(t)+v(t)}, \\
\dot{y}(t)=\frac{k_{1} x\left(t-\tau_{1}\right) v\left(t-\tau_{1}\right)}{x\left(t-\tau_{1}\right)+v\left(t-\tau_{1}\right)}-d_{2} y(t) \\
\dot{v}(t)=a y\left(t-\tau_{2}\right)-d_{3} v(t) .
\end{array}\right.
$$

In this model, $x, y$ and $v$ denote the concentration of uninfected cells, infected cells and free virus respectively. Where $\tau_{1}$ represents the delay in the production of productively infected cells and $\tau_{2}$ represents the delay in the production of virus. With the initial conditions of model (3) are given by $x(\theta)>0, y(\theta)>0$ and $v(\theta)>0$ for $\theta \in[-\rho, 0]$, where $\rho=\max \left(\tau_{1}, \tau_{2}\right)$. 


\section{Global analysis of the delay model}

\subsection{Steady states}

For our model (2), the basic reproduction number of the virus is given by

$$
R_{0}=\frac{a k_{1}}{d_{2} d_{3}} .
$$

The model (2) has two steady states:

- The disease-free steady state $E_{f}=\left(\frac{\lambda}{d_{1}}, 0,0\right)$.

- The endemic steady state

$$
E^{*}=\left(x_{1}, y_{1}, v_{1}\right),
$$

where

$$
\begin{aligned}
& x_{1}=\frac{\lambda}{d_{1}+k_{1}\left(1-\frac{1}{R_{0}}\right)}, y_{1}=\frac{\lambda k_{1}\left(1-\frac{1}{R_{0}}\right)}{d_{2}\left(d_{1}+k_{1}\left(1-\frac{1}{R_{0}}\right)\right)} \\
& \text { and } v_{1}=\frac{\lambda\left(R_{0}-1\right)}{d_{1}+k_{1}\left(1-\frac{1}{R_{0}}\right)} .
\end{aligned}
$$

\subsection{Global analysis}

In this subsection, we study the global asymptotic stability of the steady states using a suitable Lyapunov functional. For this reason, we consider the function

$$
F(x)=x-1-\ln (x) \forall x>0 .
$$

Note that $F(x)>0, \forall x>0$ and that $F(x)=0$ if and only if $x=1$. We consider the positive real numbers $x_{1}, x_{2}, \ldots, x_{n}$, then

$$
1-x_{i}+\ln x_{i}=-F\left(x_{i}\right) \leq 0, \quad \forall i=1, \ldots, n
$$

By summing for $i=1, \ldots, n$, we get:

$$
n-\sum_{i=1}^{n} x_{i}+\ln \prod_{i=1}^{n} x_{i} \leq 0,
$$

if we take $x_{i}=\frac{p_{i}}{q_{i}}$, we will have

$$
n-\sum_{i=1}^{n} \frac{p_{i}}{q_{i}}+\ln \prod_{i=1}^{n} \frac{p_{i}}{q_{i}} \leq 0,
$$

Theorem 1 If $R_{0} \leq 1$, then the disease-free steady state $E_{f}$ is globally asymptotically stable for any delays $\tau_{1}>0$ and $\tau_{2}>0$.

Proof. Let the following Lyapunov functional:

$$
\begin{aligned}
\mathcal{L}_{1}(x, y, v)= & +\frac{d_{2}}{a} v \\
& +k_{1} \int_{t-\tau_{1}}^{t} \frac{x(\xi) v(\xi)}{x(\xi)+v(\xi)} d \xi+d_{2} \int_{t-\tau_{2}}^{t} y(\xi) d \xi
\end{aligned}
$$

The time derivative is given by:

$$
\begin{aligned}
\dot{\mathcal{L}}_{1}(x, y, v) & =\dot{y}+\frac{d_{2}}{a} \dot{v} \\
& +k_{1}\left(\frac{x(t) v(t)}{x(t)+v(t)}-\frac{x\left(t-\tau_{1}\right) v\left(t-\tau_{1}\right)}{x\left(t-\tau_{1}\right)+v\left(t-\tau_{1}\right)}\right) \\
& +d_{2}\left(y(t)-y\left(t-\tau_{2}\right)\right) \\
& =\frac{k_{1} x(t) v(t)}{x(t)+v(t)}-\frac{d_{2} d_{3}}{a} v(t) \\
& \leq k_{1} v(t)-\frac{d_{2} d_{3}}{a} v(t) \\
& \leq \frac{d_{2} d_{3}}{a}\left(R_{0}-1\right) v(t) .
\end{aligned}
$$

If $R_{0}<1$; then $\dot{\mathcal{L}} \leq 0$. Moreover, $\dot{\mathcal{L}} \leq 0$ when $v=0$. The largest compact invariant is

$$
E=\left\{(x, y, v) \in R_{*}^{+} \mid v=0\right\} .
$$

According to LaSalle's invariance principle, $\lim _{+\infty} v(t)=$ 0 , the limit system of equations is:

$$
\left\{\begin{array}{l}
\dot{x}=\lambda-d_{1} x \\
\dot{y}=-d_{2} y
\end{array}\right.
$$

We define:

$$
\mathcal{L}_{1}(x, y)=\frac{1}{x_{0}}\left(x-x_{0}-x_{0} \ln \frac{x}{x_{0}}\right)+y .
$$

Since $x_{0}=\frac{\lambda}{d_{1}}$, then

$$
\dot{\mathcal{L}}(x, y)=d_{1}\left(2-\frac{x}{x_{0}}-\frac{x_{0}}{x}\right)-d_{2} y .
$$

Since the arithmetic mean is greater than or equal to the geometric mean, it follows

$$
2-\frac{x}{x_{0}}-\frac{x_{0}}{x} \leq 0
$$

Therefore $\dot{\mathcal{L}} \leq 0$ and the equality holds if $x=x_{0}$ and $y=0$, which complete the proof.

Theorem 2 The endemic steady state $E^{*}$ is globally asymptotically stable when $R_{0}>1$, for any delays $\tau_{1}>0$ and $\tau_{2}>0$.

\section{Proof.}

Let the following Lyapunov functional:

$$
\begin{aligned}
\mathcal{L}_{2}(x, y, v) & =x-x_{1}-\int_{x_{1}}^{x} \frac{d_{2} y_{1}}{\frac{k_{1} u v_{1}}{u+v_{1}}} d u+y-y_{1} \\
& -y_{1} \ln \frac{y}{y_{1}}+\frac{d_{2}}{a}\left(v-v_{1}-v_{1} \ln \frac{v}{v_{1}}\right) \\
& +k_{1} \frac{x_{1} v_{1}}{x_{1}+v_{1}} \int_{t-\tau_{1}}^{t} F\left(\frac{x(\xi) v(\xi)}{x_{1} v_{1}} \frac{x_{1}+v_{1}}{x(\xi)+v(\xi)}\right) d \xi \\
& +d_{2} y_{1} \int_{t-\tau_{2}}^{t} F\left(\frac{y(\xi)}{y_{1}}\right) d \xi .
\end{aligned}
$$


then, we have

$$
\begin{aligned}
\dot{\mathcal{L}}_{2}(x, y, v) & =-\frac{d_{1} v_{1}}{x\left(x_{1}+v_{1}\right)}\left(x-x_{1}\right)^{2} \\
& -\left(d_{2}+k_{2}\right) y_{1}\left(\frac{x\left(v-v_{1}\right)^{2}}{v_{1}\left(x+v_{1}\right)(x+v)}\right) \\
& +d_{2} y_{1}\left(4-\frac{x_{1}}{x} \frac{x+v_{1}}{x_{1}+v_{1}}-\frac{y_{1}}{y} \frac{x\left(t-\tau_{1}\right) v\left(t-\tau_{1}\right)}{x_{1} v_{1}}\right. \\
& \times \frac{x_{1}+v_{1}}{x\left(t-\tau_{1}\right)+v\left(t-\tau_{1}\right)}-\frac{y\left(t-\tau_{2}\right) v_{1}}{y_{1} v}-\frac{x+v}{x+v_{1}} \\
+ & \ln \left(\frac{x\left(t-\tau_{1}\right)+v\left(t-\tau_{1}\right)}{x v} \frac{x+v}{x\left(t-\tau_{1}\right)+v\left(t-\tau_{1}\right)}\right. \\
& \left.\left.\frac{y\left(t-\tau_{2}\right)}{y}\right)\right),
\end{aligned}
$$

Taking $p_{1}=x_{1}\left(x+v_{1}\right), p_{2}=y_{1}\left(x\left(t-\tau_{1}\right) v\left(t-\tau_{1}\right)\right)$, $p_{3}=y\left(t-\tau_{2}\right) v_{1}, p_{4}=x+v, q_{1}=x\left(x_{1}+v_{1}\right), q_{2}=$ $y\left(x_{1} v_{1}\right), q_{3}=y_{1} v, q_{4}=x+v_{1}$ and using the inequality (7) for $n=4$, we obtain

$$
\begin{aligned}
& 4-\frac{x_{1}}{x} \frac{x+v_{1}}{x_{1}+v_{1}}-\frac{y_{1}}{y} \frac{x\left(t-\tau_{1}\right) v\left(t-\tau_{1}\right)}{x_{1} v_{1}} \\
& \times \frac{x_{1}+v_{1}}{x\left(t-\tau_{1}\right)+v\left(t-\tau_{1}\right)}-\frac{y\left(t-\tau_{2}\right) v_{1}}{y_{1} v}-\frac{x+v}{x+v_{1}} \\
+ & \ln \left(\frac{x\left(t-\tau_{1}\right)+v\left(t-\tau_{1}\right)}{x v} \frac{x+v}{x\left(t-\tau_{1}\right)+v\left(t-\tau_{1}\right)}\right. \\
& \left.\frac{y\left(t-\tau_{2}\right)}{y}\right) \leq 0
\end{aligned}
$$

Therefore, $\dot{\mathcal{L}}_{2} \leq 0$. Assume that $M$ is the largest invariant set in $(x, y, v) \mid \dot{\mathcal{L}}_{2}=0$. Note that $\dot{\mathcal{L}}_{2}=0$ if and only if $x=x_{1}, y=y_{1}$ and $v=v_{1}$. Hence, $M=E^{*}$. Since $E^{*}$ exists whenever $R_{0}>1$, then by the Lyapunov-LaSalle invariance theorem $E^{*}$ is globally asymptotically stable if $R_{0}>1$.

\section{Numerical results and simulations}

In order to perform the numerical simulations, the system (1.1) will be solved numerically using the fourth order Runge-Kutta iterative scheme. The used parameter values are taken from Table 1.

Figure 1 represents the uninfected cells, the infected cells and the virus as function of time. The solid curves represent the case of $\left(\tau_{1}, \tau_{2}\right)=(5,5),(10,10)$ and $(30,30)$. For the parameters used in this figure, the basic reproduction number is $R_{0}=0.39<1$; we clearly see that the delays do not affect the global asymptotic stability behavior of the disease-free equilibrium point $E_{f}=(719.42,0,0)$. This numerical result is consistent with the theoretical result concerning the stability of $E_{f}$. Finally, Fig. 2 represents the

\begin{tabular}{|c|c|c|c|}
\hline Parameters & Meaning & Value & Reference \\
\hline$\lambda$ & $\begin{array}{l}\text { Source rate of } \\
\mathrm{CD} 4+\mathrm{T} \text { cells }\end{array}$ & {$[0,10]$} & {$[5]$} \\
\hline$k_{1}$ & $\begin{array}{l}\text { Average of in- } \\
\text { fection }\end{array}$ & {$\left[2.5 \times 10^{-4}, 0.5\right]$} & {$[3]$} \\
\hline$d_{1}$ & $\begin{array}{l}\text { Decay rate of } \\
\text { healthy cells }\end{array}$ & 0.0139 & {$[3]$} \\
\hline$d_{2}$ & $\begin{array}{l}\text { Death rate of } \\
\text { infected } \mathrm{CD} 4+ \\
\mathrm{T} \text { cells }\end{array}$ & 0.5776 & [3] \\
\hline$a$ & $\begin{array}{l}\text { The rate of } \\
\text { production } \\
\text { the virus by } \\
\text { infected CD } 4+ \\
\text { T cells }\end{array}$ & {$[2,1250]$} & [3] \\
\hline$d_{3}$ & $\begin{array}{l}\text { Clearance rate } \\
\text { of virus }\end{array}$ & {$[0.3466,2.4]$} & [3] \\
\hline
\end{tabular}
uninfected cells, the infected cells and the virus as function of time. The solid curves represent the case of $\left(\tau_{1}, \tau_{2}\right)=(5,5),(10,10)$ and $\left.(30,30)\right)$. For the parameters used in this figure, the basic reproduction number is $R_{0}=19.98>1$; we clearly see that the magnitude of the two delays does not affect the global asymptotic stability of the endemic equilibrium point
Table 1. Parameters, their symbols and default values used in the suggested HIV model
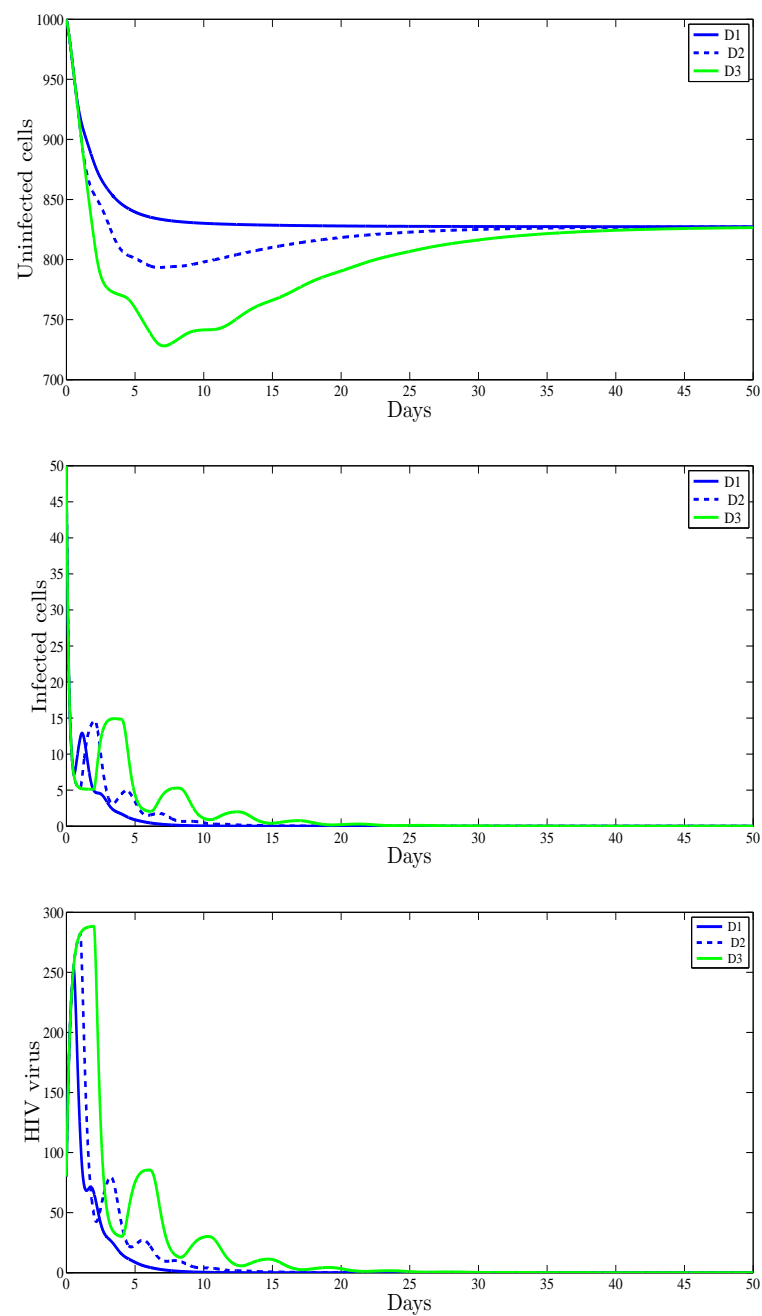

Fig. 1. The behavior of the infection dynamics for $\lambda=10$, $d_{1}=0.0139, k_{1}=0.04, d_{2}=0.5776, a=2, d_{3}=0.3466$ and $D_{1}:\left(\tau_{1}, \tau_{2}\right)=(5,5) ; D_{2}:\left(\tau_{1}, \tau_{2}\right)=(10,10) ; D_{3}:$ $\left(\tau_{1}, \tau_{2}\right)=(30,30)$.

$E^{*}=\left(214.49,172.09,2.97 \times 10^{4}, 0,0\right)$. This numerical result is consistent with the theoretical result concerning the stability of $E^{*}$. 

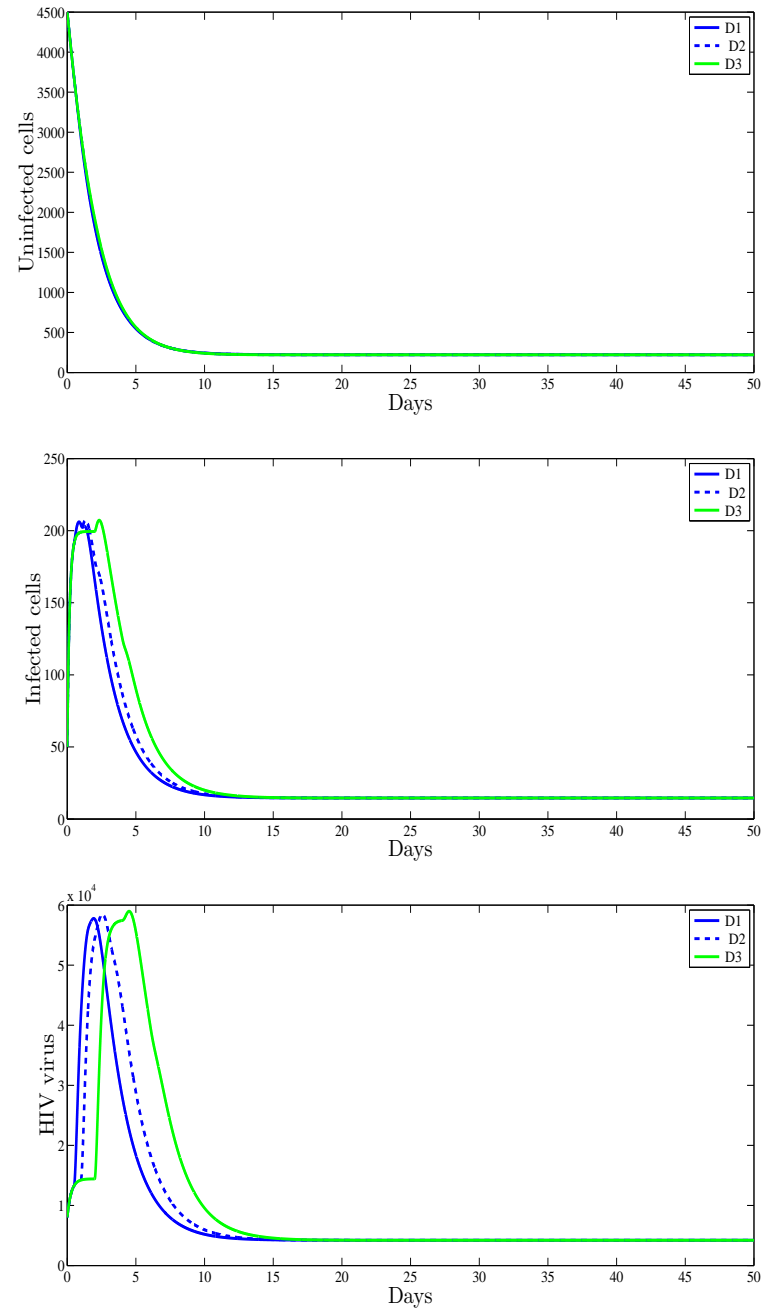

Fig. 2. The behavior of the infection dynamics for $\lambda=10$, $d_{1}=0.0139, k_{1}=0.04, d_{2}=0.5776, a=100, d_{3}=0.3466$ and $D_{1}:\left(\tau_{1}, \tau_{2}\right)=(5,5) ; D_{2}:\left(\tau_{1}, \tau_{2}\right)=(10,10) ; D_{3}:$ $\left(\tau_{1}, \tau_{2}\right)=(30,30)$.

\section{Discussion}

The HIV dynamics involving three densities of the uninfected cells, the infected cells and the HIV virus have been widely studied in different mathematical models $[2-6]$. In this work, we propose a modified viral dynamics model, using a saturated incidence function with two delays in the generation of infected cells and in the production of viruses. It was observed that the delays have a clear effect on the dynamics of the HIV infection by slowing down viral replication and reducing the amount of the infected cells.

\section{Conclusion}

In this work, we have studied the model of the HIV dynamics with time delay. The model includes a saturated rates in order to better describe the viral infection. Further, we have incorporated two delays in the model where the first delay stands for the needed time of the production of infected cells from the uninfected ones and the second delay describes the needed time for the virus production. These two delays have been included in the model to check if these delays can affect the global stability. The results show that inclusion of these delays does not influence the global dynamics of the delay model. Finally, the numerical simulations are performed in order to show the behavior of infection during the days of observation and to support the theoretical findings.

\section{References}

1. World Health Organization HIV/AIDS Key facts, (July 2014), http://www.who.int.

2. Nowak, M.A., Bonhoeffer, S., Shaw, G.M., May, R.M.(1997) Anti-viral drug treatment: Dynamics of resistance in free virus and infected cell populations. J. Theor. Biol. 184(2):203-217.

3. Sun, Q., Min, L., \& Kuang, Y. (2015) Global stability of infection-free state and endemic infection state of a modified human immunodeficiency virus infection model. IET systems biology, 9(3), 95-103.

4. Allali, K., Danane, J. and Kuang, Y., 2017. Global Analysis for an HIV Infection Model with CTL Immune Response and Infected Cells in Eclipse Phase. Applied Sciences (2076-3417), 7(8).

5. Wang, Y., Zhou, Y., Wu, J., Heffernan, J.(2009). Oscillatory viral dynamics in a delayed HIV pathogenesis model. Math. Biosci. 219, 104-112.

6. Wang, X., Wang, W. (2012) An HIV infection model based on a vectored immunoprophylaxis experiment. Journal of theoretical biology 313:127-135.

7. Q. Sun, L. Min. Dynamics Analysis and Simulation of a Modified HIV Infection Model with a Saturated Infection Rate. Computational and mathematical methods in medicine, 2014; 2014, Article ID 145162, 14 pages.

8. Hale J, Verduyn Lunel SM(1993) Introduction to functional differential equations, applied mathematical science, vol 99. Springer, New York.

9. Y. Kuang, Delay Differential Equations with Applications in Population Dynamics, Academic Press, San Diego, 1993.

10. R.V. Culshaw, S. Ruan, A delay-differential equation model of HIV infection of D4+ T-cells, Math. Biosci. 165(2000) 27-39.

11. R.V. Culshaw, S. Ruan, G. Webb, A mathematical model of cell-to-cell HIV-1 that include a time delay, J. Math.Biol. 46 (2003) 425-444.

12. J.E. Mittler, B. Markowitz, D.D. Ho, A.S. Perelson, Improved estimates for HIV-1 clearance rate and intracellular delay, AIDS 13 (1999) 1415-1417. 